\title{
Publisher Correction to: 3D-model for Active Vibration Control of Rotating Machines Mounted on Active Machine Foot Mounts Using Vibration Mode Coupling by Asymmetry
}

Ulrich Werner ${ }^{1}$

Published online: 9 November 2020

○ Krishtel eMaging Solutions Private Limited 2020

\section{Publisher correction to: Journal of Vibration Engineering \& Technologies https://doi. org/10.1007/s42417-020-00252-z}

The publication of this article unfortunately contained mistakes due to publisher's error. Please see the corrected parts below:

$$
\begin{array}{ll}
\mathbf{A}_{\mathbf{s t}}=\left[\begin{array}{cc}
\mathbf{0}_{6} & \mathbf{I}_{6} \\
-\mathbf{M}^{-1} \cdot \mathbf{C}-\mathbf{M}^{-1} \cdot(\mathbf{D}+\mathbf{G})
\end{array}\right] ; & \mathbf{B}_{\mathbf{s t}}=\left[\begin{array}{c}
\mathbf{0}_{6} \\
\mathbf{M}^{-1}
\end{array}\right] \\
\mathbf{C}_{\mathbf{s t}}=\left[\begin{array}{cc}
\mathbf{I}_{6} & \mathbf{0}_{6} \\
\mathbf{0}_{6} & \mathbf{I}_{6} \\
-\mathbf{M}^{-1} \cdot \mathbf{C}-\mathbf{M}^{-1} \cdot(\mathbf{D}+\mathbf{G})
\end{array}\right] ; & \mathbf{D}_{\mathbf{s t}}=\left[\begin{array}{c}
\mathbf{0}_{6} \\
\mathbf{0}_{6} \\
\mathbf{M}^{-1}
\end{array}\right]
\end{array}
$$

to set to the zero vector $\mathbf{E}(s)=\mathbf{0}$. Finally, the poles can be

$$
\begin{aligned}
\mathbf{G}_{\gamma, \mathbf{\kappa}}(j \omega)= & \frac{\mathbf{Y}_{\gamma, \mathbf{\kappa}}(j \omega)}{E_{\mathbf{\kappa}}(j \omega)}=\mathbf{G}_{\gamma}(j \omega) \cdot \mathbf{P}_{\mathbf{e}, \mathbf{\kappa}} \\
= & {\left[\mathbf{I}_{18}+\left(\mathbf{C}_{\mathbf{s t}} \cdot\left(\mathbf{I}_{12} \cdot j \omega-\mathbf{A}_{\mathbf{s t}}\right)^{-1} \cdot \mathbf{B}_{\mathbf{s t}}+\mathbf{D}_{\mathbf{s t}}\right) \cdot \mathbf{T}_{\mathbf{s t}, \gamma}(j \omega)\right]^{-1} } \\
& \cdot\left[\mathbf{C}_{\mathbf{s t}} \cdot\left(\mathbf{I}_{12} \cdot j \omega-\mathbf{A}_{\mathbf{s t}}\right)^{-1} \cdot \mathbf{B}_{\mathbf{s t}}+\mathbf{D}_{\mathbf{s t}}\right] \cdot \mathbf{P}_{\mathbf{e}, \mathbf{\kappa}}
\end{aligned}
$$

$\tilde{\mathbf{M}} \cdot \ddot{\mathbf{q}}+(\tilde{\mathbf{D}}+\mathbf{G}) \cdot \dot{\mathbf{q}}+\tilde{\mathbf{C}} \cdot \mathbf{q}=\mathbf{0}$

$\left[\tilde{\mathbf{M}} \cdot \lambda^{2}+(\tilde{\mathbf{D}}+\mathbf{G}) \cdot \lambda+\tilde{\mathbf{C}}\right] \cdot \hat{\mathbf{q}}=\mathbf{0}$ sources of excitation and the blocks $v_{-} z b D, v_{-} z b N, v_{-} y b D$.

with $\mathbf{T}_{\mathbf{z}}(s), \mathbf{T}_{\mathbf{v}}(s), \mathbf{T}_{\mathbf{a}}(s) \in \mathbb{C}^{6 \times 6}$ and $\mathbf{T}_{0}(s)=\mathbf{0}_{6} \in \mathbb{R}^{6 \times 6}$. set to the zero vector $\mathbf{E}(s)=\mathbf{0}$.

$\left[\mathbf{I}_{12} \cdot s-\mathbf{A}_{\mathbf{s t}}+\mathbf{B}_{\mathbf{s t}} \cdot \mathbf{T}_{\mathbf{s t}, \boldsymbol{\gamma}} \cdot\left[\mathbf{I}_{18}+\mathbf{D}_{\mathbf{s t}} \cdot \mathbf{T}_{\mathbf{s t}, \gamma}\right]^{-1} \cdot \mathbf{C}_{\mathbf{s t}}\right] \cdot \mathbf{X}(s)=\mathbf{0}$

The original article has been corrected.

Publisher's Note Springer Nature remains neutral with regard to jurisdictional claims in published maps and institutional affiliations.

The original article can be found online at https://doi.org/10.1007/ s42417-020-00252-z.

Ulrich Werner

ulrich.werner@th-nuernberg.de

Nuremberg Tech, Georg Simon Ohm University of Applied Sciences Nuremberg, Faculty EFI, 90489 Nuremberg, Germany 